\title{
ANÁLISIS DE FACTIBILIDAD DEL DISEÑO DE UN SISTEMA SOLAR FOTOVOLTAICO EN LA ESCUELA CAMPO 45 DEL CORREGIMIENTO CENTRO DE LA CIUDAD DE BARRANCABERMEJA
}

\author{
FEASIBILITY ANALYSIS OF A SOLAR PHOTOVOLTAIC SYSTEM DESIGN
}

IN THE SCHOOL CAMPO 45 OF THE CENTER RURAL ZONE OF THE CITY

OF BARRANCABERMEJA

\section{Jefferson Alexander Montañez}

Est. Ingeniería Ambiental, Universidad Nacional Abierta y a Distancia Perfil e Institución

jamontanezl@unadvirtual.edu.co

\section{Jineth Lorena Vargas}

Est. Ingeniería Ambiental, Universidad Nacional Abierta y a Distancia jlvargasb@unadvirtual.edu.co

\section{Eliana Marcela Trujillo}

Ingeniera Ambiental, Universidad Nacional Abierta y a Distancia eliana.marcela9@hotmail.com

\section{Sergio Suárez Palacios}

Docente ocasional -Cadena de Formación Ambiental, Grupo de Conservación, Bioprospección y Desarrollo Sostenible, Universidad Nacional Abierta y a Distancia

sergio.suarez@unad.edu.co

\section{Resumen}

La energía solar fotovoltaica se caracteriza por generar electricidad a partir de fotoceldas que se exponen a una radiación solar, su importancia se ve enfocada en el aumento significativo de la eficiencia energética y el mínimo impacto ambiental que se puede presentar en comparación con otros sistemas de generación.

DOI: https://doi.org/10.22490/26653176.2785 


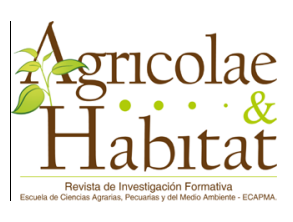

Las variables más significativas que necesita un sistema solar fotovoltaico para la escuela Campo 45 del municipio de Barrancabermeja fueron de gran importancia a la hora de definir la factibilidad de diseño y dimensionamiento de una potencial instalación de dicho sistema, por lo cual, se realizó una búsqueda exhaustiva de los parámetros climatológicos, un pre-dimensionamiento del sistema en espacio físico libre de instalación, así como, un análisis de costo aproximado para su ejecución. Para un mejor acercamiento, se obtuvo de dicho análisis que en la zona se pueden obtener un promedio mensual de $155 \mathrm{kWh} / \mathrm{m}^{2}$, lo que permitiría un aprovechamiento adecuado de energía eléctrica en la escuela si se colocan aproximadamente 5 paneles solares que soporten la demanda de energía eléctrica mensual aproximada de $649 \mathrm{kWh} / \mathrm{mes}$; de igual forma, se realizó una revisión de sistemas a utilizar con paneles que permite ver las diferentes características que pueden ser necesarias a la hora de un buen funcionamiento del sistema, además, se propone un seguimiento en el mantenimiento, teniendo en cuenta las condiciones del lugar. De este proyecto se puede concluir que en la escuela o en la zona de influencia directa, es factible realizar un diseño y una implementación de un sistema solar fotovoltaico que permita el abastecimiento de la necesidad energética de la población.

Palabras clave: Calculadora solar; Demanda energética; Eficiencia energética; Panel solar.

\section{Abstract}

Photovoltaic solar energy is characterized by generating electricity from cells or photocells that are exposed to solar radiation, its importance is focused on the significant increase in energy efficiency and the minimum environmental impact that may occur compared to other generation systems.

The most significant variables a photovoltaic solar system needs for Campo 45 school in the Barrancabermeja city, were of great importance when defining the feasibility of design and size of a potential installation of the system, for this reason, an exhaustive search of the climate parameters, a pre-sizing of the system in free physical space of installation, as well as, an approximate cost analysis for its execution, was carried out. For a better approach, it was found from this analysis that, in the area, a monthly average of $155 \mathrm{kWh} / \mathrm{m} 2$ of energy can be obtained, which would allow an adequate use of electric power in the school if approximately 5 solar panels are placed to support the demand 


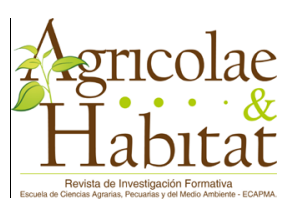

of approximately $649 \mathrm{kWh}$ / electric power per month; Likewise, a systems review was made, using panels that allow to see the different characteristics which are necessary to have a good functioning of the system; in addition, maintenance monitoring is proposed, taking into account the conditions of the place. From this project we can conclude that it is feasible to design and implement a photovoltaic solar system that allows the supply of the population's energy needs in the school or in the direct influence area.

Keywords: Energy demand; Energy efficiency; Solar calculator; Solar panel.

\section{Introducción}

La implementación de un sistema de energía fotovoltaica ha sido un éxito en muchos proyectos realizados en la actualidad, por ello, el estudio minucioso de algunos proyectos exitosos que se han desarrollado en Colombia, como es el proyecto de diseño de un sistema solar fotovoltaico autónomo para una institución educativa rural en el municipio de Páez, Boyacá (Mesa L, Sanabria C \& Pérez W, 2016), por otro lado, el proyecto de instalación de un sistema de energía solar en una vereda de Firavitoba, con el objetivo de bombear agua para el acueducto rural (El tiempo, 2016) y por último, el primer proyecto de energía renovable en Barrancabermeja, el cual consistió en la implementación de paneles solares, con el objetivo de abastecer de energía eléctrica a las oficinas de la empresa electrificadora (Dígame, 2018), todos ellos, indicando que el uso y adecuación de este tipo de sistema es viable.

El estudio de variables para el diseño de un sistema solar fotovoltaico en la escuela campo 45 del corregimiento centro de la ciudad de Barrancabermeja, se hace a partir de un trabajo realizado en el curso de energía solar térmica y fotovoltaica en el primer semestre del año 2018, con el fin de mejorar a futuro la calidad de vida y educación para los niños que estudian allí, debido a la carencia de energía eléctrica por falta de recursos económicos para cubrir este servicio. De acuerdo a la 
información geográfica obtenida para el estudio de este sistema de energía, se pudo determinar que Barrancabermeja está ubicada en el Magdalena medio, contando con una temperatura promedio de $29^{\circ} \mathrm{C}$ en promedio, y con varias horas de sol que favorecen en gran dimensión la implementación de este proyecto, ya que su potencia y/o energía solar presente en esta zona, puede suplir los requerimientos que necesitan dichos paneles.

\section{Metodología}

La demanda de energía eléctrica del edificio se encuentra a partir de un cálculo sencillo que interpreta la trazabilidad en tiempo de los recibos de la luz, esto con el fin de determinar específicamente un promedio de energía que necesita la escuela para su funcionamiento.

Es importante destacar, que la versión gratuita del programa solarius PV (ACCA software, s.f.), nos pudo arrojar unos resultados pertinentes de la energía solar disponible en el lugar del proyecto, así mismo, el uso de un calculador solar web (Calculationsolar.com, s.f.) en la que se pudo obtener el azimut solar. Por otro lado, se realizó un presupuesto básico teniendo en cuenta valores promedio de los elementos encontrados en suministradores de los equipos del sistema, el cual permitió, dar a conocer un valor aproximado de la posible instalación.

\section{Resultados y discusión}

\section{Energía solar disponible en el sitio exacto del proyecto}

Según la ubicación exacta del sitio a implementar el proyecto (Escuela campo 45 corregimiento centro Barrancabermeja, Santander) y de acuerdo a datos suministrados por el programa solarius PV (Ver figura 1) podemos determinar que la irradiación anual en esta zona es de $1865,02 \mathrm{kWh} / \mathrm{m}^{2}$, esto quiere decir que al mes se está obteniendo una irradiación de $155,41 \mathrm{kWh} / \mathrm{m}^{2}$, además otro dato que podemos 
determinar son los meses en donde se recibe más radiación diaria, los cuales son febrero y agosto, en donde se realizará más provecho solar, supliendo adecuadamente la energía requerida por el sistema.

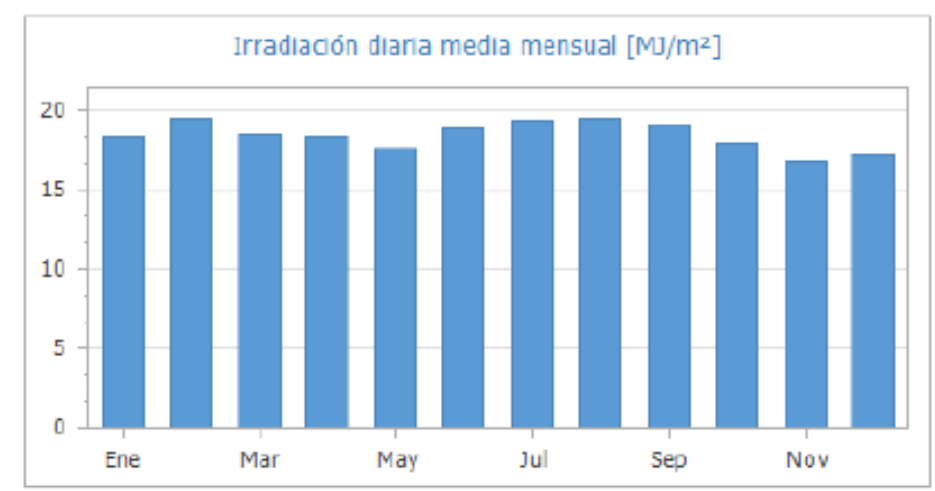

Figura 1. Energía solar disponible en el corregimiento de Barrancabermeja, Santander. Fuente: Solarius PV

\section{Abastecimiento de energía eléctrica a la población}

De acuerdo al consumo promedio de energía que requiere la población benéfica en este proyecto podemos concluir que (Ver Tabla 1) la cantidad de kWh/hora por mes es de 649, es decir que diarios se necesitan aproximadamente $21,63 \mathrm{kWh}$ para un adecuado desempeño de sistema solar fotovoltaico.

Determinando la población consumidora la cual es 50 personas incluyendo estudiantes y directivos, podemos determinar que cada persona necesita de $12,98 \mathrm{kWh}$ al mes para suplir sus necesidades. 
Tabla 1. Consumo promedio de energía de los habitantes de la escuela campo 45 del corregimiento centro, Barrancabermeja.

\begin{tabular}{|c|c|}
\hline VARIABLE & RESULTADO \\
\hline $\begin{array}{l}\text { kWh/hora } x \text { mes } \\
(k W h)\end{array}$ & $649 \mathrm{kWh}$ \\
\hline kWh/hora x día $(\mathrm{kWh})$ & $21,63 \mathrm{kWh}$ \\
\hline Población consumidora & 50 personas \\
\hline kWh/habitante & $12,98 \mathrm{kWh}$ \\
\hline
\end{tabular}

\section{Irradiación solar}

El promedio de la radiación solar o potencia solar diaria mensual obtenida en el corregimiento centro de la ciudad de Barrancabermeja (zona a implementar el proyecto) es de 5,31 kWh/día (Ver Tabla 2), lo que permitió dar a conocer que es una buena zona para montar una instalación solar fotovoltaicas y que, a partir de los gastos energéticos de la escuela se obtendrá el número exacto de la cantidad de paneles a usar.

Tabla 2. Irradiación diaria media mensual [ $\mathrm{kWh} / \mathrm{m} 2]$ en el corregimiento de Barrancabermeja. Fuente: Solarius PV

\begin{tabular}{|l|l|l|l|l|l|l|l|l|l|l|l|l|}
\hline Ene & Feb & Mar Abr May Jun & Jul & Ago & Sep & Oct & Nov Dic \\
\hline 5,34 & 5,57 & 5,18 & 5,03 & 4,74 & 5,04 & 5,19 & 5,30 & 5,30 & 5,09 & 4,85 & 5,00 \\
\hline
\end{tabular}

FUENTE: SOLARIUS PV 


\section{Funcionamiento potencial del sistema}

Se encuentran y se proponen 4 tipos de paneles solares de diferentes características de producción de energía, con los parámetros ideales de funcionamiento, además de sus propiedades físicas y condiciones normales de operación.

Tabla 3. Características técnicas de 4 tipos de paneles solares fotovoltaicas.

\begin{tabular}{|c|c|c|c|c|}
\hline & $\begin{array}{l}\text { Panasonic } \\
\text { Sanyo } \\
\text { VBHN295 } \\
2855 J 46\end{array}$ & $\begin{array}{l}\text { Kyocera- } \\
\text { KK270P- } \\
\text { 3CB3CG }\end{array}$ & $\begin{array}{l}\text { Sunpower } \\
\text { X21-335blk- } \\
345\end{array}$ & $\begin{array}{lr}\text { Trina } & \text { Solar } \\
\text { Honey } & \text { TSM } \\
\text { DD05A_08II }\end{array}$ \\
\hline Potencia (W) & 295W & $270 W$ & $345 \mathrm{~W}$ & $290 \mathrm{~W}$ \\
\hline Eficiencia (\%) & $19.1 \%$ & $16.4 \%$ & $21.5 \%$ & $17.7 \%$ \\
\hline Tolerancia (\%) & $0 /+10 \%$ & $-3 /+5 \%$ & $0 /+5 \%$ & $0 /+5 \%$ \\
\hline NOCT $\left({ }^{\circ} \mathrm{C}\right)$ & $44^{\circ} \mathrm{C}$ & $45^{\circ} \mathrm{C}$ & $41.5^{\circ} \mathrm{C}$ & $44^{\circ} \mathrm{C}(+-2 \%)$ \\
\hline Peso (Kg) & $18 \mathrm{~kg}$ & $19 \mathrm{~kg}$ & $18.6 \mathrm{Kg}$ & $18.6 \mathrm{Kg}$ \\
\hline Dimensiones & $1463 \times 1053 \mathrm{~mm}$ & $1662 \times 990 \mathrm{~mm}$ & $\begin{array}{l}1559 \times 1046 \\
\mathrm{~mm}\end{array}$ & $1650 \times 992 \mathrm{~mm}$ \\
\hline Vidrio & $\begin{array}{l}\text { Templado con } \\
\text { revestimiento } \\
\text { antirreflectante }\end{array}$ & $\begin{array}{l}\text { Templado con } \\
\text { revestimiento } \\
\text { antirreflectante }\end{array}$ & $\begin{array}{l}\text { Templado } \\
\text { Antirreflejo } \\
\text { Transmisión }\end{array}$ & $\begin{array}{l}\text { Templado Alta } \\
\text { Transparencia }\end{array}$ \\
\hline Marco & $\begin{array}{l}\text { Aluminio } \\
\text { anodizado negro }\end{array}$ & $\begin{array}{l}\text { Aluminio } \\
\text { anodizado Plata }\end{array}$ & $\begin{array}{l}\text { Aluminio } \\
\text { anodizado } \\
\text { Clase } 1\end{array}$ & $\begin{array}{l}\text { Aluminio } \\
\text { anodizado negro }\end{array}$ \\
\hline 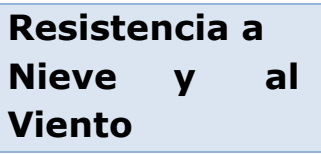 & $2400 \mathrm{~Pa}$ & $2400 \mathrm{~Pa}$ & $2400 \mathrm{~Pa}$ & $2400 \mathrm{~Pa}$ \\
\hline $\begin{array}{l}\text { Garantía del } \\
\text { Producto }\end{array}$ & 15 años & 10 años & 25 años & 10 años \\
\hline $\begin{array}{l}\text { Garantía del } \\
\text { Rendimiento }\end{array}$ & $\begin{array}{l}10 \text { años / 90\% } \\
25 \text { años / 80\% }\end{array}$ & $\begin{array}{l}10 \text { años / 90\% } \\
25 \text { años / 80\% }\end{array}$ & $\begin{array}{l}5 \text { años/ } 90 \% \\
5 \text { a } 25 \text { años / } \\
0.4 \% \text { año }\end{array}$ & $\begin{array}{l}25 \text { años con } \\
\text { rendimiento } \\
\text { lineal en } \\
\text { Potencia }\end{array}$ \\
\hline
\end{tabular}

Fuente: (Mappt solar,2017) 


\section{Planos y revisión física del sistema}

Para este sistema se propone una posible ubicación de los paneles solares sobre cubierta donde se puede encontrar el suficiente espacio, así como, un lugar donde ningún tipo de elemento obstruya el paso de radiación directa sobre el sistema, lo que permite un desarrollo efectivo del sistema sin necesidad de hacer un control de sombras.

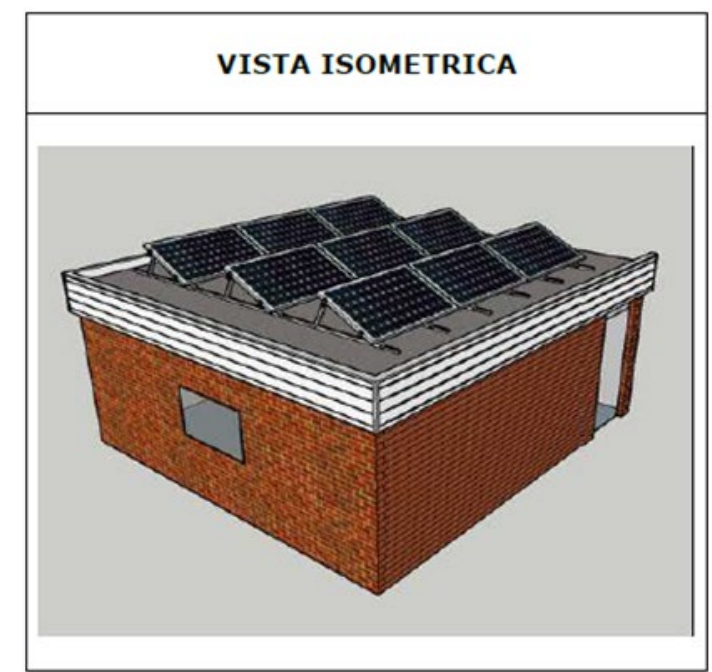

Figura 2. Vista isométrica de la propuesta de instalación del sistema solar fotovoltaico sobre cubierta.

\section{Costos y presupuesto}

Tabla 4. Análisis general de costos de instalación.

\begin{tabular}{|c|c|c|c|c|c|}
\hline & Descripción & $\begin{array}{l}\text { Unida } \\
\text { d }\end{array}$ & $\begin{array}{l}\text { Cantida } \\
\text { d }\end{array}$ & $\begin{array}{l}\text { Precio } \\
\text { unitario }\end{array}$ & $\begin{array}{l}\text { Precio } \\
\text { parcial }\end{array}$ \\
\hline \multirow[t]{5}{*}{$\begin{array}{l}\text { Equipos y } \\
\text { materiales }\end{array}$} & Panel solar & EA & 9 & $\begin{array}{l}\$ \\
760.000\end{array}$ & $\begin{array}{l}\$ \\
6.840 .000\end{array}$ \\
\hline & Regulador 3W & EA & 1 & $\begin{array}{l}\$ \\
240.000\end{array}$ & $\begin{array}{l}\$ \\
240.000\end{array}$ \\
\hline & $\begin{array}{l}\text { Baterias } 12 \mathrm{~V}- \\
220 \mathrm{Ah}\end{array}$ & EA & 4 & $\begin{array}{l}\$ \\
1.545 .000\end{array}$ & $\begin{array}{l}\$ \\
6.180 .000\end{array}$ \\
\hline & Inversor $3 \mathrm{~kW}$ & EA & 1 & $\begin{array}{l}\$ \\
1.870 .000\end{array}$ & $\begin{array}{l}\$ \\
1.870 .000\end{array}$ \\
\hline & $\begin{array}{l}\text { Instalación } \\
\text { eléctrica }\end{array}$ & GL & 1 & $\begin{array}{l}\$ \\
2.000 .000\end{array}$ & $\begin{array}{l}\$ \\
2.000 .000\end{array}$ \\
\hline
\end{tabular}




\begin{tabular}{|c|c|c|c|c|c|}
\hline & $\begin{array}{lr}\text { Valor } & \text { total } \\
\text { equipos } & y \\
\text { materiales } & \end{array}$ & & & & $\begin{array}{l}\$ \\
17.130 .000\end{array}$ \\
\hline \multirow[t]{7}{*}{$\begin{array}{l}\text { Montaje e } \\
\text { instalación }\end{array}$} & Paneles solares & EA & 9 & $\begin{array}{l}\$ \\
120.000\end{array}$ & $\begin{array}{l}\$ \\
1.080 .000\end{array}$ \\
\hline & Regulador 3W & EA & 1 & $\begin{array}{l}\$ \\
535.000\end{array}$ & $\begin{array}{l}\$ \\
535.000\end{array}$ \\
\hline & $\begin{array}{l}\text { Baterias } 12 \mathrm{~V}- \\
220 \mathrm{Ah}\end{array}$ & EA & 4 & $\begin{array}{l}\$ \\
80.000\end{array}$ & $\begin{array}{l}\$ \\
320.000\end{array}$ \\
\hline & Inversor $3 \mathrm{~kW}$ & EA & 1 & $\begin{array}{l}\$ \\
1.250 .000\end{array}$ & $\begin{array}{l}\$ \\
1.250 .000\end{array}$ \\
\hline & $\begin{array}{l}\text { Instalación } \\
\text { eléctrica }\end{array}$ & $\mathrm{GL}$ & 1 & $\begin{array}{l}\$ \\
1.500 .000\end{array}$ & $\begin{array}{l}\$ \\
1.500 .000\end{array}$ \\
\hline & $\begin{array}{lr}\text { Valor } & \text { total } \\
\text { montaje } & \text { y } \\
\text { construcción } & \end{array}$ & & & & $\begin{array}{l}\$ \\
4.685 .000\end{array}$ \\
\hline & Gran total & & & & $\begin{array}{l}\$ \\
21.815 .000\end{array}$ \\
\hline
\end{tabular}

\section{Conclusiones}

Con la iniciativa y desarrollo de este proyecto, se encontró la problemática que se vive en la escuela de campo 45 en el corregimiento el centro en la ciudad de Barrancabermeja, este centro educativo se encuentra retirado del casco urbano, lo que hace que el acceso de energía eléctrica convencional sea limitado, al poder ejecutar este diseño del proyecto de energía renovable, será una fuente energética sostenible para una mejor calidad de vida y educación para los estudiantes de esta institución.

El diseño del proceso de generación de energía eléctrica a partir de paneles fotovoltaicos de la escuela veredal campo 45, se plantea como un sistema directo de conversión energética, la dirigen y orientan hacia un sistema de acumulación, con el fin de ser aprovechada en este plantel educativo. 
El sistema diseñado para la escuela veredal campo 45, ofrece resultados de bienestar a los estudiantes y la escuela, el cual se beneficiarán de bombeo de agua potable, servicio en la sala de informática sin interrupciones, iluminación y aprovechamiento en el uso de herramientas que necesitan de energía eléctrica.

Las horas de sol de la ciudad de Barrancabermeja permiten llevar a cabo con mayor seguridad el desarrollo de este proyecto, pues la mayoría de los meses se encuentra en verano la ciudad, son muy pocos los meses lluviosos que se dan y por consiguiente días despejados y sin radiación difusa que disminuya la eficiencia del sistema.

Desde una perspectiva económica, la respuesta es que los costos son elevados por concepto de electricidad y que representan el mayor gasto energético en la escuela. A la luz de este hecho, la continua baja en los precios de los sistemas solares y la adopción de modelos de financiación innovadores ofrecen la oportunidad de reducir drásticamente su gasto de energía, siendo el periodo de retorno de inversión más corto y obteniendo un sistema de generación de energía que pueda abastecer la necesidad durante un tiempo prolongado.

\section{Referencias Bibliograficas}

ACCA software. (s.f.). Software cálculo de instalación fotovoltaica. Recuperado de https://www.accasoftware.com/es/softwarecalculo-instalacion-fotovoltaica

BOYACÁ SIE7E DÍAS. (13 de Julio de 2016). Energía solar, solución en vereda de Firavitoba. (E. Tiempo, Ed.) Boyacá, Colombia. Recuperado de https://www.eltiempo.com/archivo/documento/CMS-16643731

Calculationsolar.com. (s.f.). Recuperado de http://calculationsolar.com/es/ 
Digame.com.co. (23 de Febrero de 2018). Primer proyecto de energía renovable en Barrancabermeja. Barrancabermeja, Santander, Colombia. Recuperado de http://www.digame.com.co/primerproyecto-de-energia-renovable-en-barrancabermeja-2/

Dinamov. (13 de Mayo de 2015). Granja Solar Fotovoltaica 121,4 kW Tunja Boyacá. Tunja, Boyacá, Colombia. Recuperado de http://www.dinamov.co/ver blog/3 granja solar fotovoltaica 11 $4 \mathrm{kw}$ tunja boyaca

Mesa, L. A., Sanabria, C. A., \& Pérez, W. J. (19 de Octubre de 2016). Diseño de un sistema solar fotovoltaico autónomo parra una institución educativa rural en el municipio de Páez - Boyacá. Paéz, Boyacá, Colombia. Recuperado de http://fcbi.unillanos.edu.co/cici/Articulos/CICI 2016 paper 148.p $\underline{\mathrm{df}}$

Mpptsolar. (2017). Los mejores paneles solares del mundo. Recuperado de https://www.mpptsolar.com/es/mejores-paneles-solares.html

Torres Piamba, L. J. (25 de mayo de 2017). CONTROL, SEGUIMIENTO Y MANTENIMIENTO EN PANELES SOLARES. Recuperado de https://www.youtube.com/watch?v=t4ifgtj2wJ8

Vega Colmenares, J. Y. (15 de Septiembre de 2017). Granja de energía solar: nuevo proyecto de inversión en Santander. Barrancabermeja, Santander, Colombia. Recuperado de http://barrancabermeja.extra.com.co/noticias/local/granja-deenergia-solar-nuevo-proyecto-de-inversion-en-santa-348092 